\title{
An Experimental Microstructural Characterization of High- quality, Load-preserved Fabric 1-D Consolidated Kaolinite Samples
}

\author{
Jun Kang Chow ${ }^{1}$, Zhaofeng $\mathrm{Li}^{1}$, and Yu-Hsing Wang ${ }^{1 *}$ \\ ${ }^{1}$ Hong Kong University of Science and Technology, Department of Civil and Environmental Engineering, HKSAR, China
}

\begin{abstract}
This paper describes a microstructural characterizations of high-quality, load-preserved fabric 1D consolidated kaolinite samples, which covers from the beginning stage of clay sample preparation to the final stage of the microstructural analyses. To achieve this goal, a tailor-made oedometer is produced using the 3-D printing technique. First, a uniform kaolinite sample is prepared from a slurry state and then positioned into the 3-D printed oedometer for 1-D consolidation tests. Then, together with the applied loadings, the whole oedometer containing the consolidated kaolinite sample is submerged into the liquid nitrogen. This aims for preparing the dry sample by freeze drying, and at the same time, preserving the fabric associations for the subsequent microstructural characterizations. Afterwards, the sample is cut in half while frozen. An observation plane along the centre with the morphological information preserved is used for the scanning electron microscopy (SEM) analyses, and the remaining section is undergone the mercury intrusion porosimetry to obtain complementary information on the pore-size distribution. By ensuring the position and orientation of the SEM images taken, the number of SEM images, as well as the amount of particles and voids identified are maximized to enhance the statistical representation of the analysed results. In each sample, at least 3000 particles are identified, and the voids are segmented using proper binary images, of which their irregular shapes are further described using an equivalent ellipse. Fabric tensors are used to quantify the directional behaviour of the voids and particles. In addition, the shape evolution of the pores is examined to further understand the associated deformation mechanism. These comprehensive analyses provides quantitative evidences that the loading response of clay under 1-D consolidation is mainly governed by the inter-aggregate pores.
\end{abstract}

\section{Introduction}

The microstructural characteristics of clays are closely related to the macroscopic properties under different conditions, such as the loading history, stress state and pore fluid properties $[1,2]$. Therefore, tremendous effort has been devoted to gain insights about the interaction and arrangement among different fabrics, including particles, aggregates and pores. However, some underlying problems encountered at different stages of microstructural characterizations remain unsolved. For instance, the rebound effect, due to unloading clay samples before drying, is unavoidable and has been considered to cause only minimal disturbance on the clay fabric [3, 4]. The results obtained from different measurement techniques also may not be representative and comparable, as the same sample is not used normally in all the measurements. In addition, most of the time, quantitative analyses of the clay samples are rarely reported. Hence, a working pipeline is proposed in this study to tackle all the aforementioned challenges so as to comprehensively characterize the microstructural behaviour of clay based on high-quality, load-preserved and orientation-known one-dimensional (1-D) samples.

The paper is organized as follows. It begins with experimental details of all the stages in sample preparations, followed by the microstructural analyses with the mercury intrusion porosimetry (MIP) and scanning electron microscopy (SEM) techniques. The difficulties encountered in each stage and the corresponding solutions adopted in this study are delineated. Then, the experimental results are presented, including the deformation characteristics, changes in the pore-size distribution and fabric arrangements of clay samples due to consolidation.

\section{Proposed pipeline}

In this study, Speswhite kaolin powder (from Imerys Minerals Ltd., UK) was used. Kaolinite samples were prepared, followed by the microstructural analyses and characterizations, according to the procedures described in Chow and Wang [5] and Chow et al. [6].

\footnotetext{
* Corresponding author: ceyhwang@ust.hk
} 


\subsection{Slurry consolidation and sample trimming}

The kaolinite powder was first mixed with deionized water, in an amount about two times the liquid limit (LL) $(\mathrm{LL}=65 \%$, as measured by Yu et al. [7]), i.e., $\sim 1.3 \mathrm{~kg}$ of water was mixed with $1 \mathrm{~kg}$ of dry kaolinite powder. The mixed slurry was consolidated in a tailor-made, long consolidometer of $80 \mathrm{~mm}$ wide and $400 \mathrm{~mm}$ high, with a vertical pressure of $\sim 60 \mathrm{kPa}$, under double drainage condition. Following slurry consolidation, the kaolinite sediment was carefully taken out. As shown in Fig. 1, only the central part of the sediment, which was believed to be less affected by the boundary conditions, was used. Several cylindrical samples, with $\sim 8 \mathrm{~mm}$ in height and $12 \mathrm{~mm}$ in diameter, were trimmed from this sediment, using a sharp trimmer.

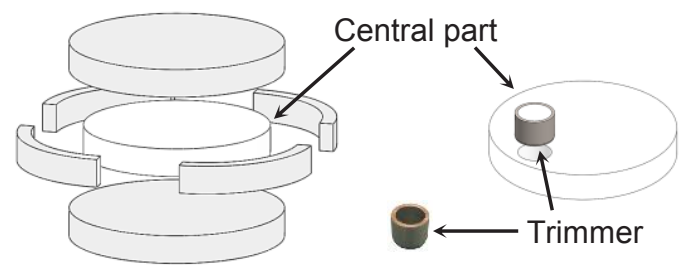

Fig. 1. Selection of sample, where only the central part is used for trimming small kaolinite sample for 1-D consolidation test.

\subsection{1-D consolidation tests with the tailor-made 3D-printed oedometer}

Dried samples are essential for implementing the MIP and SEM analyses. The sample has to be unloaded prior to the removal of water, but such an action is susceptible to alter the original structure of clay samples. In order to preserve the fabric arrangement at the original loading state during rapid freezing (one of the steps in removing water), as shown in Fig. 2, a 3D-printed oedometer was invented to carry out the 1-D consolidation tests; more details are provided in Chow and Wang [5].

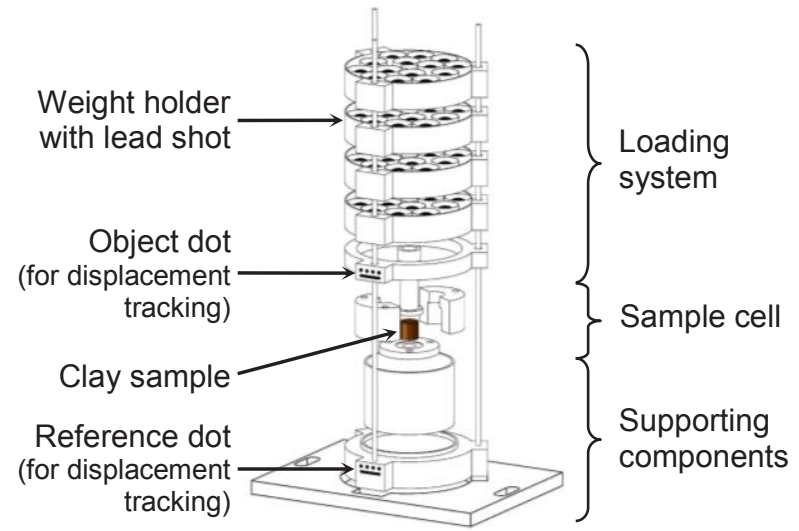

Fig. 2. The tailor-made 3D-printed oedometer.

As shown in Fig. 3, Particle Image Velocimetry (PIV) and close-range photogrammetry techniques [8] were used to determine the sample deformation during 1-D consolidation tests. A digital camera equipped with a timer controller was used to continuously capture highresolution images $(5184 \times 3456$ pixels $)$ for the subsequent PIV analyses. Four 1-D consolidation tests, up to maximum vertical stress of $\sigma^{\prime}{ }_{v}=30,100,200$ and
$250 \mathrm{kPa}$, were carried out, according to ASTM D2435. In each test, loading was applied for four times, with an increment ratio of $\sim 1$, up to the maximum $\sigma^{\prime}{ }_{v}$. For each loading increment, the consolidation time was $\sim 12$

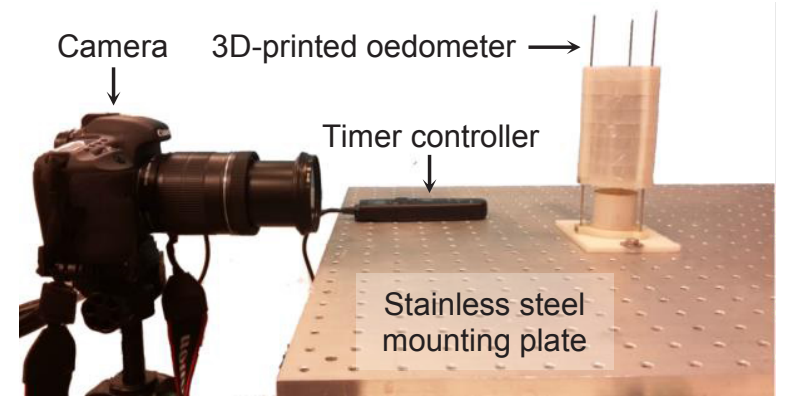

hours.

Fig. 3. Experimental setup for 1-D consolidation test using the 3D-printed oedometer.

\subsection{Specimen preparation for microstructural analyses}

Together with the applied loads, the whole 3D-printed oedometer containing the consolidated kaolinite sample was submerged into liquid nitrogen for rapid freezing of pore water. This aimed to prepare sample for subsequent freeze drying, and at the same time, to avoid the undesirable unloading effect. Then, still in a frozen conditions, freeze cutting was performed to cut the frozen kaolinite sample in half. A vertical surface was created at the centre for SEM analyses.

Right after cutting, as shown in Fig. 4, the frozen kaolinite sample was carefully placed in a 3D-printed covered square container to maintain a known orientation and to protect the sample from any external disturbance. The cover was removed upon freeze drying, and this process lasted for $\sim 48$ hours to remove water via sublimation so as to minimize the capillary effects.

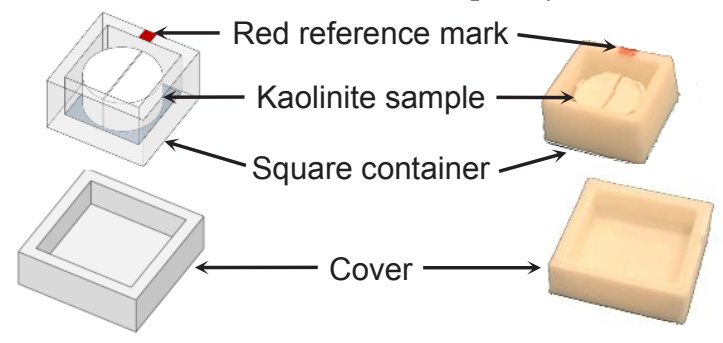

Fig. 4. The 3D-printed covered square container.

\subsection{Microstructural analyses}

Using identical sample throughout all microstructural analyses is crucial so that the results obtained are representative and comparable. Therefore, similar specimens from the same loaded sample, i.e., the two halves samples, were respectively used for the MIP and SEM analyses. The MIP analysis was conducted to measure the pore-size distribution of the kaolinite samples. Then, the SEM imaging was performed with to obtain high-resolution SEM images, along the vertical observation plane, with optimized accelerating voltage, magnification factor and working distance. Note that a reference dot was marked to track the specimen 
orientation. In order to enhance the statistical representation of the analyses, for each kaolinite sample, sufficiently large numbers (i.e., $20 \sim 30$ ) of SEM images of size $9.14 \mu \mathrm{m} \times 6.85 \mu \mathrm{m}(1280 \times 960$ pixels $)$ were taken.

\section{Experimental results}

In this section, the consolidation behaviour of kaolinite samples, i.e., the deformation characteristics, pore-size distribution and microstructural responses, are presented.

\subsection{Deformation characteristics of 1-D consolidation}

As shown in Fig. 5, the responses at different consolidation pressures are consistent, suggesting that similar properties are exhibited by all the samples.

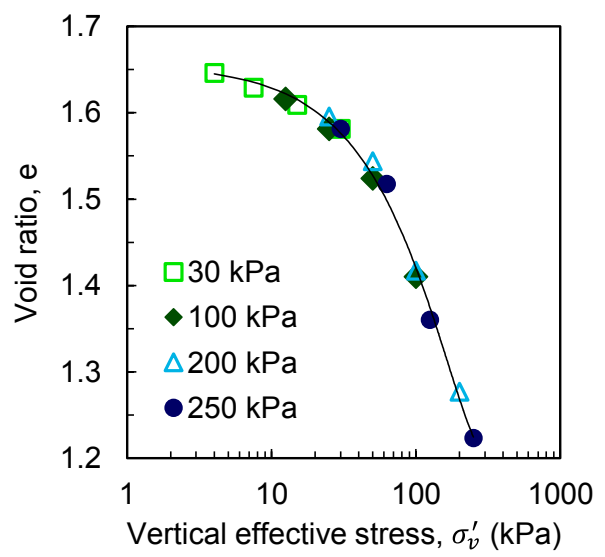

Fig. 5. The consolidation curves of kaolinite samples at different applied loadings.

\subsection{Changes in pore-size distribution in response to 1-D consolidation}

Fig. 6 presents the pore-size distributions of kaolinite samples, based on the MIP analyses. For comparison, the sample after slurry-consolidation, referred to as the initial state, is also presented. The pores are revealed to gradually compress with an increase of $\sigma_{v}^{\prime}$, indicating by the shifting of the peak of the curve towards the smaller size. The estimated boundary of intra- and interaggregate pores is $\sim 0.14 \mu \mathrm{m}$, based on the findings $[2,7$, 9] that the volume of intra-aggregate pores remains almost unchanged upon consolidation.

\subsection{Fabric characterizations based on the SEM images}

Fig. 7 presents a SEM image of kaolinite particles. The kaolinite particles, which appeared as narrow grey strips in the SEM images, were manually identified. At least $\sim 3000$ particles were annotated to provide representative result. Next, the raw SEM photographs were converted into binary images, followed by performing threshold segmentation to annotate the voids. The region-based method [10] was then used to appropriately describe the features of the extracted voids, using a fitted equivalent ellipse.

Fabric tensors were used to quantify the directional distribution of both the particles and voids [11]. The major-axis length of the microstructural entities was chosen as the weighting parameter to reflect the anisotropy behaviour. The fabric tensors computed from the 2-D SEM images can be further described using the major principal direction $(\theta)$ and anisotropy intensity factor $(\alpha)$, i.e., the difference between the major and minor principal components.

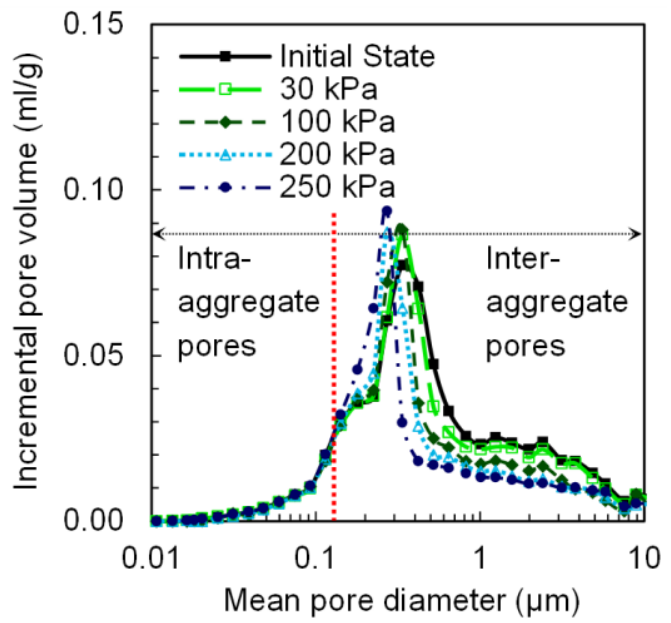

Fig. 6. The pore-size distribution of kaolinite samples after 1-D consolidation subjected to different applied loadings.

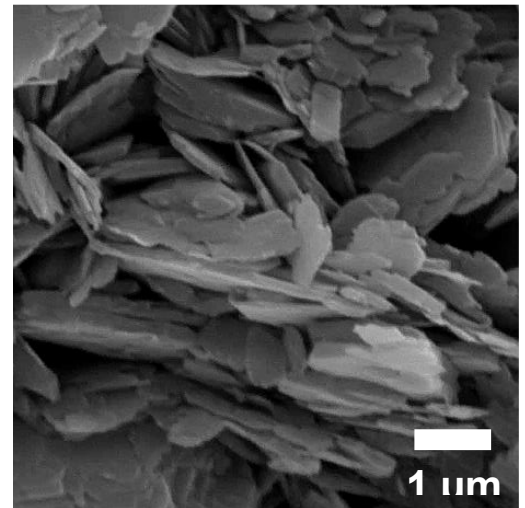

Fig. 7. SEM image of kaolinite particles.

\subsubsection{The directional distribution of particles and voids, and their associated fabric tensors}

Fig. 8 summarizes the results quantified with the particle-based fabric tensors (i.e., $\theta_{p}$ and $\alpha_{p}$ ) subjected to different consolidation pressures $\left(\sigma^{\prime}{ }_{v}\right)$. As shown in Fig. 8 , as $\sigma_{v}^{\prime}$ increases, the particle orientation is gradually towards the horizontal plane, representing by the higher $\alpha_{p}$ along $\theta_{p} \approx 0^{\circ}$. This provide additional evidence to the experimental findings by other researchers $[6,9,12]$, where the particles converge along the plane perpendicular to the loading direction as $\sigma^{\prime}{ }_{v}$ increases.

Fig. 9 presents the summary of the results computed from the void-based fabric tensors (i.e., $\theta_{v}$ and $\alpha_{v}$ ) at different $\sigma^{\prime}{ }_{v}$. Coincidentally, like the behavior of particles, the void also converges to the horizontal direction, representing by higher $\alpha \mathrm{v}$ around $\theta_{v} \approx 0^{\circ}$. 


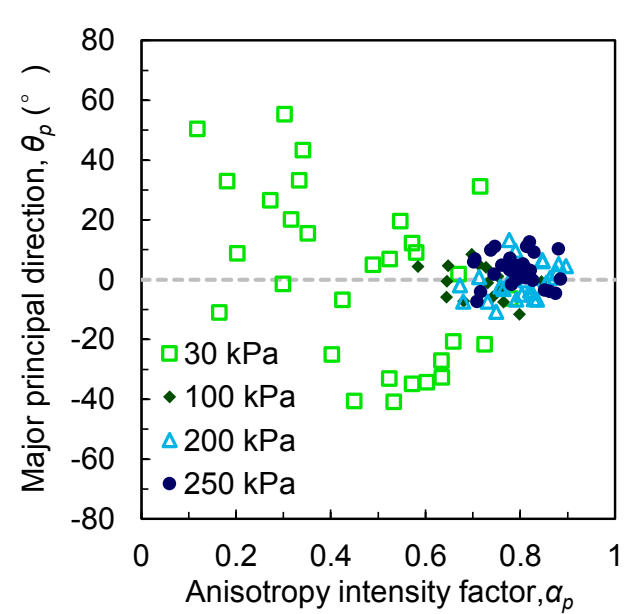

Fig. 8. A summary of the major principal direction and anisotropy intensity factor computed from the particle-based fabric tensors subjected to different consolidation pressures.

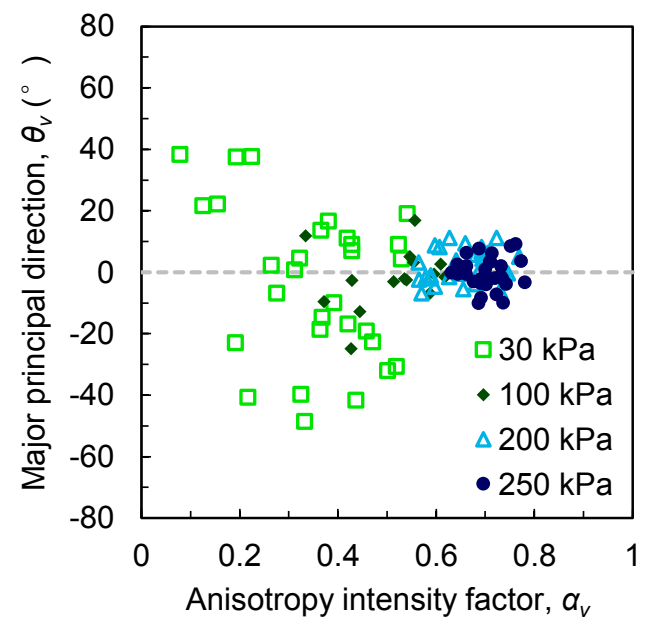

Fig. 9. A summary of the major principal direction and anisotropy intensity factor computed from the void-based fabric tensors subjected to different consolidation pressures.

\subsubsection{Shape evolution of voids}

In addition to the changes of pore-orientation provided by the fabric tensor analyses, the pore-shape features upon consolidation was examined. To distinguish the responses of inter- and intra-aggregate pores [6], the number fraction $(n)$, defined as the ratio of the number of the specific type of pores observed to the total number of pores, was introduced. The area fraction $(A)$ was adopted as well in terms of the cross-sectional area.

Fig. 10 summarizes the pore features of kaolinite sample after consolidation at $\sigma^{\prime}{ }_{v}=30$ and $250 \mathrm{kPa}$. As shown in the figure, although the number of intraaggregate pores is larger (i.e, $n_{\text {intra }}>n_{\text {inter }}$ ), the crosssectional area occupied by the inter-aggregate pores is found to be much greater (i.e., $A_{\text {inter }} \gg A_{\text {intra }}$ ). This verifies that the inter-aggregate pores are dominant in the loading response, and therefore the void response, as observed in Fig. 8, is mainly contributed by the interaggregate pores.

Further, the average elongation factor $(E)$, measured within intervals of $10^{\circ}$ of void orientation, was introduced to quantify the changes in the shape of interaggregate pores, as shown in Eq. 1.

$$
E=\sum\left(l_{\text {minor }} / l_{\text {major }}\right) / N
$$

where $l_{\text {minor }}$ and $l_{\text {major }}$ respectively are the minor- and major-axis lengths of the equivalent ellipses used to describe the voids, and $N$ is the total number of pores. The evolution of $E$ is also presented in Fig. 10. For the pores that are aligned horizontally $\left(\sim 0^{\circ}\right)$, the applied loading further compresses and elongates them, representing by decrease in $E$ as $\sigma_{v}^{\prime}$ increases. On the other hand, for the pores that are aligned vertically (i.e, $90^{\circ}$ or $\left.90^{\circ}\right), E$ increases with an increase in $\sigma_{v}^{\prime}$, indicating that the applied loading gradually compresses the flocculated structure, accompanied with rearrangement of aggregates of particles.
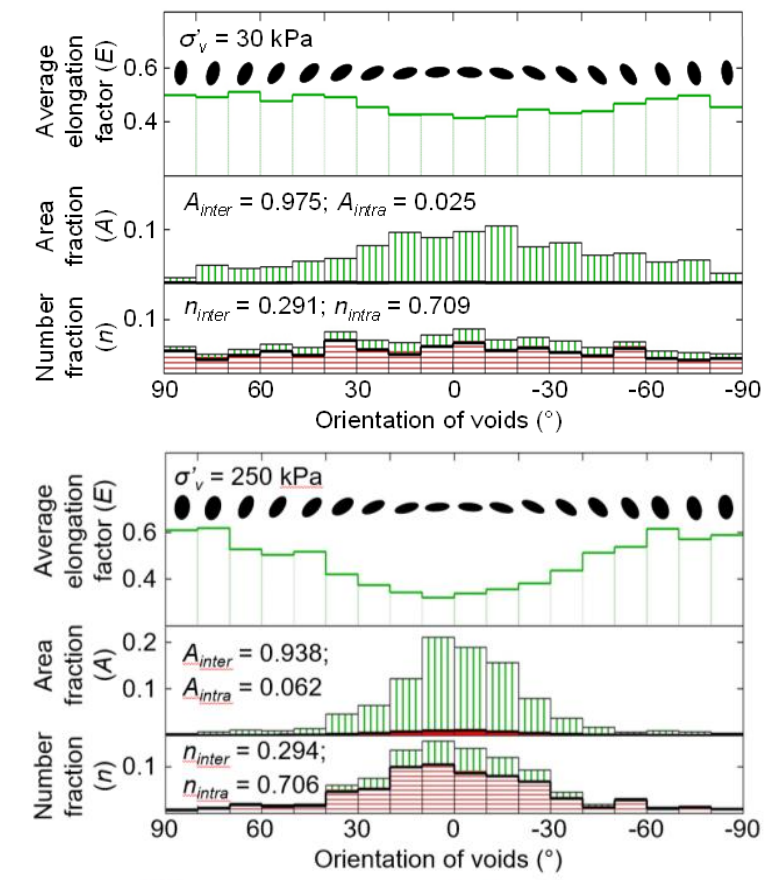

IIIII Inter-aggregate pores $\square$ Intra-aggregate pores

Fig. 10. A summary of the pore features of kaolinite samples subjected to consolidation pressures of 30 and $250 \mathrm{kPa}$.

\section{Conclusions}

In this study, a pipeline is proposed in order to comprehensively characterize the microstructural behavior of kaolinite samples upon consolidation. Highquality, load-preserved and orientation-known samples are prepared so that the analysed results obtained are representative and comparable. The comprehensive analyses provides quantitative evidences that the loading response of clay under 1-D consolidation is mainly governed by the inter-aggregate pores. This pipeline is applicable to other geotechnical testing, and the characterization results could be further enhanced and automated with advanced algorithmic methods, such as machine learning techniques.

\section{References}

1. J.C. Santamarina, K.A. Klein, M.A. Fam, Soils and Waves: Particulate Materials Behavior, Characterizations and Process Monitoring (2001). 
2. P. Delage, G. Lefebvre, Can. Geotech. J. 21(1), 2135 (1984).

3. H. Cetin, Eng. Geol. 73, 1-11 (2004).

4. M. Hattab, J.M. Fleureau, Géotechnique 60(5), 323331 (2010).

5. J.K. Chow, Y.H. Wang, Geotech. Testing J. 40(5), 891-905 (2017).

6. J.K. Chow, Z. Li, Y.H. Wang, Eng. Geol. 28, 22-33 (2019).

7. C.Y. Yu, J.K. Chow, Y.H. Wang, Eng. Geol. 202, 122-131 (2016).

8. D.J. White, W.A. Take, M.D. Bolton, Géotechnique 53(7), 619-631 (2003)

9. Y.H. Wang, D. Xu, J. Geotech. Geoenviron. Eng. 133(7), 793-801 (2007).

10. K.F., Mulchrone, K.R., Choudhury, J. Struct. Geol. 26, 145-153 (2004).

11. P. Fu, Y.F. Dafalias, Int. J. Solids Struct. 63, 68-81 (2015).

12. P.Y. Hicher, H. Wahyudi, D. Tessier, Mech. Cohesive-Frict. Mater. 5(5), 341-371 (2000). 